\title{
Video Article \\ Human Placental and Decidual Organ Cultures to Study Infections at the Maternal-fetal Interface
}

\author{
Gabrielle A. Rizzuto ${ }^{1,2}$, Mirhan Kapidzic ${ }^{3}$, Matthew Gormley ${ }^{3}$, Anna I. Bakardjiev ${ }^{1,4,5}$ \\ ${ }^{1}$ Benioff Children's Hospital \\ ${ }^{2}$ Department of Pathology, University of California, San Francisco \\ ${ }^{3}$ Center for Reproductive Sciences and Department of Obstetrics, University of California, San Francisco \\ ${ }^{4}$ Program in Microbial Pathogenesis and Host Defense, University of California, San Francisco \\ ${ }^{5}$ Biomedical Sciences Program, University of California, San Francisco
}

Correspondence to: Anna I. Bakardjiev at Anna.Bakardjiev@ucsf.edu

URL: https://www.jove.com/video/54237

DOI: doi:10.3791/54237

Keywords: Immunology, Issue 113, Human placenta, human decidua, Listeria monocytogenes, pathogenesis, organ culture

Date Published: $7 / 21 / 2016$

Citation: Rizzuto, G.A., Kapidzic, M., Gormley, M., Bakardjiev, A.I. Human Placental and Decidual Organ Cultures to Study Infections at the Maternal-fetal Interface. J. Vis. Exp. (113), e54237, doi:10.3791/54237 (2016).

\section{Abstract}

The placenta shows a large degree of interspecies anatomic variability. To best understand biology and pathophysiology of the human placenta, it is imperative to design experiments using human cells and tissues. An advantage of organ culture is maintenance of three-dimensional (3D) structural organization and extracellular matrix. The goal of the method described here is successful establishment of ex vivo human gestational tissue organ cultures and their healthy culture maintenance for $72-96 \mathrm{hr}$. The protocol details the immediate processing of researchconsented, placental and decidual specimens fresh from the operating suite. These are abundant specimens that would otherwise be discarded. Detailed instructions on the sterile collection of these samples, including morphologic details on how to select appropriate tissues to establish 3D organ cultures, is provided. Placental villous and decidual tissues are microdissected into $2-3 \mathrm{~mm}^{3}$ pieces and placed separately on matrixlined transwell filters and cultured for several days. Villous and decidual organ cultures are well suited for the study of human host-pathogen interaction. As compared to other model organisms, these human cultures are particularly advantageous to examine mechanism of infection for pathogens that demonstrate variable patterns of host specificity. As an example, we demonstrate infection of placental and decidual organ cultures with the clinically relevant, facultative intracellular bacterial pathogen Listeria monocytogenes.

\section{Video Link}

The video component of this article can be found at https://www.jove.com/video/54237/

\section{Introduction}

Infection and inflammation at the maternal-fetal interface represents a major source of morbidity and mortality in women and children.

Understanding how pathogens infect these tissues is critical for the development of novel strategies to prevent and treat diseases such as preterm labor and fetal death. However, high interspecies variability of the maternal-fetal interface complicates experimental investigation. Further, most microbial pathogens show host specificity, thus many animal models cannot fully recapitulate human infectious diseases. Certain organisms (Haemophilus influenzae, Salmonella typhi, Vibrio cholera, and numerous others) are strictly pathogenic in humans, while others, such as Listeria monocytogenes, show a more intermediate level of host specificity ${ }^{1}$. Host specificity has been documented in many aspects of pathogenesis, including colonization ${ }^{2}$, dissemination ${ }^{3}$, immune evasion ${ }^{4}$, and nutrient acquisition ${ }^{5}$. Thus, it is of utmost importance to select optimal host model systems.

Composed of fetal cells and maternal blood, the placenta is an organ that rapidly develops over the course of gestation ${ }^{6}$. In simplest terms, the human placenta is constructed of fetal "villous" tree-like structures bathed in maternal blood. Gas and nutrient exchange occurs across specialized fetal cell layers called trophoblasts that line the surface of the villous trees. The maternal uterine mucosal lining, termed endometrium in the non-pregnant female, transforms structurally and functionally into the decidua to accommodate the fetoplacental unit. Villous trees are anchored into the uterus by extravillous trophoblasts (EVT) that migrate from anchoring cell columns into the decidua. The maternal-fetal interface, therefore, consists of decidua and placenta.

The organ culture technique described here has been used to examine where and how clinically relevant human pathogens, including $L$. monocytogenes and Toxoplasma gondii, cross the placental barrier ${ }^{2,7}$. These ex vivo organ cultures replicate in vivo tissue architecture, and are arguably physiologically highly relevant model systems for human placentation. Additional culture techniques include whole organ explants, organ slices, and tissue or stem cell organoids embedded in 3D-matrix. For details on these options, please refer to a comprehensive recent review $^{8}$. Please note that this protocol is for the separate culture of decidua and placental villi. For studying the interaction between placental 
cells and decidual cells, a co-culture technique may be preferred. We refer the interested reader to the previously described villous-decidual coculture technique, used by researchers studying trophoblast-mediated decidual vascular remodeling ${ }^{9-11}$.

\section{Protocol}

Experiments were conducted according to the principles expressed in the Declaration of Helsinki. This study was approved by the Institutional Review Board at the University of California, San Francisco (CHR\# 11-05530). All patients provided written informed consent for the collection of samples and subsequent analysis. Tissue was collected as de-identified specimens.

NOTE: The researcher must obtain approval from their institutional human subjects review board. The exclusion of specimens based upon gestational history or placental anomalies will vary according to the needs of any specific study. NOTE: Appropriate safety conditions (biosafety level 2 ) should be utilized for all steps in this protocol ${ }^{12}$.

\section{Preparation, Prior to Collection Day}

1. Autoclave strainers/forceps for collection, and scissors/forceps for micro-dissection.

2. Prepare adequate volume (500 $\mathrm{ml}$ per specimen) of Wash buffer (refer to Table $\mathbf{1}$ for recipe).

3. Prepare adequate volume $(100 \mathrm{ml}$ per specimen) of $0.22 \mu \mathrm{M}$ sterile-filtered Collection media (refer to Table 1 for recipe).

4. Aliquot Extracellular Matrix (ECM, refer to Table of Reagents for details) for long-term storage.

5. Store ECM in solid form at $-20^{\circ} \mathrm{C}$. For best performance, avoid repeat freeze thaw. Thaw bottle to a viscous solution at $4{ }^{\circ} \mathrm{C}$, and prepare 300 $\mu \mathrm{l}$ aliquots in the cold room with chilled pipette tips. Store aliquots at $-20^{\circ} \mathrm{C}$.

\section{Collection of Fresh Tissue}

CAUTION: When working with fresh human tissue, researchers must follow Universal Precautions (OSHA) for preventing transmission of Bloodborne Pathogens, and must have received proper institutional training. Proper personal protective equipment, including gloves, eye protection, and lab coats are necessary.

1. Transport autoclaved-strainers (one per specimen), autoclaved-forceps, Wash buffer (carboy), Collection media (25 ml per specimen in a 50 $\mathrm{ml}$ conical tube), spray bottle, $10 \%$ bleach, $70 \%$ ethanol, tissue collection tray, sharpie, and ice bucket/cooler to hospital/clinic. NOTE: Specimens are received in a research-designated space located in a room close to the operating suite. A sterile tissue culture hood is not required, but collection should occur expeditiously and the researcher should appropriately sanitize surfaces, as described below.

2. Place carboy of Wash buffer adjacent to the sink, and spray spigot with $10 \%$ bleach and $70 \%$ ethanol. Place glass tissue collection tray on the light source (light pad or light box), and sanitize with $10 \%$ bleach and $70 \%$ ethanol. Allow to air dry.

3. Have the clinician carry specimens from the operating suite to the specimen preparation room. At the sink, pour the specimen atop sterile hand-held strainer, rinse tissue several times in Wash buffer, and transfer entire specimen in buffer to sanitized glass tray atop light source. NOTE: Assessment of tissue by the clinician is of highest priority, thus the following steps are only performed after the clinician has verbally indicated that he/she has finished the evaluation.

4. Use sterile forceps to select placental villi and decidua (Figures $\mathbf{2 A}$ and $\mathbf{3 A}$ ) for collection, and transfer to separate $50 \mathrm{ml}$ conical tubes. Label the tubes with gestational age.

NOTE: Preferred gestational ages are less than 9 weeks and less than 16 weeks for placenta and decidua, respectively. At the institution, only a fraction of each specimen is procured for research purposes, as adequate tissue must remain for clinical pathologic diagnosis.

5. Store and transport specimen on ice to the research lab.

6. To collect more than one specimen, sanitize the glass tray as described above with $10 \%$ bleach and $70 \%$ ethanol between the collection of each specimen.

\section{Preparation of Organ Culture Plates}

NOTE: The following steps should be performed using sterile technique in a tissue culture hood. It is ideal for the dissecting microscope to be located in a sterile field. However, this is not absolutely necessary as long as micro-dissection is performed expeditiously, and instruments are dipped frequently in $70 \%$ ethanol.

1. Thaw ECM vial(s) on ice. Dilute the ECM 1:1 with ice-cold Collection media, mix by pipetting. Pipette slowly to avoid introducing bubbles. Each well of a 6 -well tissue culture plate requires $100 \mu \mathrm{l}$ of this suspension and will accommodate 3-4 organ cultures.

2. Place transwell inserts (pore size $0.4 \mu \mathrm{M}$ ) into each well of 6-well tissue culture plate.

3. Coat each transwell insert with a thin layer $(100 \mu \mathrm{l})$ of the $\mathrm{ECM} /$ media suspension.

4. Place the plate on ice and immediately proceed to establishment of organ cultures. Alternatively, prepare the plate prior to tissue collection, in which case wrap it in para-film and store at $4{ }^{\circ} \mathrm{C}$ for later use. Storage longer than $6 \mathrm{hr}$ is not recommended as the ECM will dry out.

\section{Establishment of Organ Cultures}

NOTE: This step describes how to place decidual organ cultures and villous organ cultures in separate transwells. The tissues are not in contact. Researchers interested in a co-culture technique where decidua and villi are in direct contact with each other, can refer to prior literature ${ }^{9,10}$.

1. Rinse specimens twice in Collection media, and centrifuge at $1,000 \times \mathrm{g}$ between each rinse. Transfer tissue to a sterile petri dish and place on the stage of a dissecting microscope. Keep 6-well plate on ice adjacent to the microscope.

2. Micro-dissect tissue with sterile springer dissecting scissors and forceps, to establish 2-3 mm villous organ cultures (Figure 2A). 
NOTE: Villous organ cultures are small, well-vascularized "trees" with 2 or 3 branches, and with prominent extravillous trophoblast (EVT) cell columns $^{11}$. It is important to utilize only villous tissue from $<9$ week first trimester specimens, as invasion of extravillous trophoblasts into ECM is most pronounced at these young ages ${ }^{13}$.

3. Select well-vascularized villi with prominent EVT cell columns. EVT cell columns are visualized as bulbous, "fuzzy", "fluffy" ends (refer to arrowheads in Figure 2A).

4. Use sterile forceps to transfer 3 or 4 villous trees into each transwell. Adjust branches so tree is positioned flat atop ECM and separate clumped branches.

5. Micro-dissect decidual tissue with sterile springer dissecting scissors and forceps, to establish $3 \mathrm{~mm}^{3}$ decidual organ cultures (Figure $\mathbf{3 A}$ ). NOTE: Specimens contain two distinct types of decidual tissues, decidua parietalis and decidua capsularis (left and right tissue fragments, respectively, Figure 3A).

6. Trim with scissors to create $3 \mathrm{~mm}^{3}$ pieces of decidua parietalis. Decidua capsularis is not used for these cultures. Use forceps to tease away any clotted maternal blood.

7. Use sterile forceps to transfer 3-4 pieces of decidua into each transwell.

8. Add $1 \mathrm{ml}$ of Collection media to bottom of the well. Incubate plate overnight at $37^{\circ} \mathrm{C}$ in $5 \% \mathrm{CO}_{2}$

9. For experiments where it is important to mimic normal first trimester placental oxygen tension, maintain villous organ cultures in relative hypoxia $\left(3 \% \mathrm{O}_{2}\right)$.

NOTE: Laboratory options include small hypoxia incubator chambers that fit inside existing incubators, or a tri-gas incubator that introduces external $\mathrm{N}_{2}$ gas to lower oxygen concentration.

10. Add $1 \mathrm{ml}$ of Villous or Decidual medium (Table 1) to top of transwell after 12-16 hr of culture. NOTE: The absence of media during the first overnight in culture allows the extravillous trophoblast cell columns to invade (villous culture), and for the tissue (both villous and decidual) to become securely embedded in ECM. In our experience, decidual organ cultures remain viable for $72 \mathrm{hr}$ and villous organ cultures for $96 \mathrm{hr}$. We recommend experimental endpoints that fall within this timeframe. See Table 1 for composition of Villous and Decidual medium. Villous medium has a high percentage of FBS, while Decidual medium is supplemented with pregnancy hormones (progesterone, 17ß-estradiol) that maintain the decidualized state (Table 1).

\section{Preparation of L. monocytogenes Cultures}

NOTE: For detailed protocol on the long-term storage of $L$. monocytogenes, and the culture and growth of this organism in brain heart infusion $(\mathrm{BHI})$ broth and agar, please refer to Wang et al. ${ }^{14}$

1. Pick a single $L$. monocytogenes colony from streaked $\mathrm{BHI}$ agar plate and inoculate $3 \mathrm{ml}$ of $\mathrm{BHI}$ broth.

2. Incubate the $L$. monocytogenes culture overnight ( $16 \mathrm{hr}$ ), in a slanting position, at $30^{\circ} \mathrm{C}$. NOTE: These culture conditions allow bacterial growth to reach stationary phase. L. monocytogenes is flagellated at $30^{\circ} \mathrm{C}$, which increases host cell invasion ${ }^{15}$.

\section{Infection of Organ Cultures with L. monocytogenes}

1. Warm PBS and Villous or Decidual medium (Table 1) to $37^{\circ} \mathrm{C}$.

2. Use sterile tweezers to remove any organ culture pieces that did not invade into the ECM, and are therefore floating in the well.

3. Carefully aspirate media from bottom of transwell. Rinse upper and lower transwells twice by gently pipetting $1 \mathrm{ml}$ of warm PBS into well, and carefully aspirating in between. Gently pipette $1 \mathrm{ml}$ of antibiotic-free Villous or Decidual media to upper and lower transwell. Be careful not to disturb the organ culture.

4. Let the tissue incubate in antibiotic-free media for at least an $\mathrm{hr}$ to ensure that antibiotics have been removed. NOTE: At stationary phase the density of the L. monocytogenes culture is approximately $1 \times 10^{9}$ colony forming units (CFU) per ml. The number of bacteria used to infect organ cultures should be empirically determined to meet experimental needs.

5. For wild type $L$. monocytogenes routine infection experiments show robust invasion with a 1:50 dilution of the overnight $\left(4 \times 10^{7} L\right.$. monocytogenes in $1 \mathrm{ml}$ of media per well). Resuspend the appropriate number of bacteria in antibiotic-free Villous or Decidual medium

6. Aspirate media from top of transwells. Gently add $1 \mathrm{ml}$ of inoculum. Incubate plates at $37{ }^{\circ} \mathrm{C}$ in $5 \% \mathrm{CO}_{2}$ to allow for bacterial invasion.

7. Remove extracellular bacteria by aspirating media from upper and lower transwells. Rinse wells twice with warm PBS. Add $1 \mathrm{ml}$ of Villous or Decidual media supplemented with $50 \mathrm{\mu g} \mathrm{ml}^{-1}$ Gentamicin. Incubate plates at $37^{\circ} \mathrm{C}$ in $5 \% \mathrm{CO}_{2}$.

NOTE: $L$. monocytogenes is a facultative intracellular bacterium that can grow in extracellular medium, grow inside of cells, and spread from cell-to-cell without accessing the extracellular space. Gentamicin has a bactericidal effect on extracellular $L$. monocytogenes, and thus allows measurement of intracellular $L$. monocytogenes growth and spread through the organ culture 16 .

NOTE: Reproducible L. monocytogenes infection of villous and decidua organ cultures occurs with $5 \mathrm{hr}$ incubation times. The time of incubation before addition of gentamicin can be modified based on experimental needs and the ability of the organism to invade the tissue. To better understand the anatomic sites most vulnerable to infection, shorter incubation times can be used ${ }^{2}$.

8. Maintain plates at $37{ }^{\circ} \mathrm{C}$ in $5 \% \mathrm{CO}_{2}$ for the duration of the experiment. Change whole media in each well daily.

NOTE: In our experience, L. monocytogenes-infected decidual organ cultures remain viable for $48 \mathrm{hr}$ and villous organ cultures for $72 \mathrm{hr}$.

\section{Harvest of Organ Cultures for Histopathology}

1. Prepare a fresh solution of $4 \%$ paraformaldehyde by diluting $10 \mathrm{ml}$ of a $16 \%$ stock ampoule in $30 \mathrm{ml} \mathrm{PBS}$. Store $4 \%$ paraformaldehyde at 4 ${ }^{\circ} \mathrm{C}$, protected from light, and use within one week. Bring to room temperature prior to use.

2. Aspirate media from upper and lower transwell. Gently rinse wells twice with PBS at room temperature. Gently add $1 \mathrm{ml} 4 \%$ paraformaldehyde to top transwell to completely cover the organ culture.

3. Incubate in $4 \%$ paraformaldehyde at room temperature for 20 min. Avoid longer incubation times as this might lead to over-fixation of the tissue. 
4. Aspirate paraformaldehyde and rinse wells twice with PBS at room temperature. Embed the fixed organ cultures in OCT medium, freeze, and section on a cryostat ("fixed frozen"). In general, fixed frozen tissue is easier to section than frozen tissue without prior fixation. NOTE: For complete protocol on OCT embedding and frozen sectioning, please access relevant sections from published protocols ${ }^{17,18}$ Depending on experimental needs and available laboratory equipment and storage, tissue may instead be paraffin-embedded and sectioned on a microtome ${ }^{19}$.

5. Further prepare tissue slides for routine Hematoxylin \& Eosin staining, immunofluorescence, or immunohistochemistry ${ }^{18,20,21}$.

\section{Representative Results}

Figure 1 (modified from Zeldovich \& Bakardjiev ${ }^{22}$ ) diagrams the relevant placental and uterine anatomy. Figure 2 shows representative gross and histologic images of placental villi, while Figure 3 demonstrates decidual tissue.

This protocol first describes the collection of fresh placental and decidual tissue at the hospital or clinic. The collected specimen is a mixture of fetal placental (villi) and maternal uterine components (decidua). After rinsing with buffer containing broad-spectrum antibiotics and antifungals, the entire specimen is inspected by eye using a light box. Villi and decidua are placed in separate tubes and transported on ice to the laboratory. In the laboratory, a dissecting microscope is used to appreciate the fine morphologic features of healthy tissue. Representative photographs of villous (Figure 2A) and decidual tissues (Figure 3A) that were acquired with a dissecting microscope with two external gooseneck light sources are shown for reference.

Villous organ cultures are generated from first trimester specimens. Cultures consist of small terminal villous trees with 2-6 branches. It is important to dissect villous branches that terminate in extravillous trophoblast columns and are well-vascularized. These features are seen as "fluffy" or "fuzzy" branch ends (arrowheads), and the faint pink-to-red linear hue that courses through the branches of the tree on the left in Figure 2A. In contrast, vasculature and extravillous trophoblasts are not readily visualized for the tree on the right of this image, which would be suboptimal for culture. Over the first day of culture, the extravillous trophoblasts migrate into the ECM, thus anchoring the villous trees into the matrix on the transwell.

Decidual organ cultures are generated from first and early second trimester specimens. The entire uterine lining is transformed into decidua very shortly after implantation. More specifically, decidua basalis defines the uterine mucosa directly underlying the placenta/implantation site, decidua capsularis defines mucosa overlying the fetus, and decidua parietalis refers to the remainder of the uterine lining. Visualization under a dissecting microscope shows that the early gestational specimen consists largely of decidua parietalis and decidua capsularis (left and right tissue fragments, respectively, in Figure 3A). The decidua capsularis is easily distinguished by its thin, membranous nature. For organ cultures, decidua parietalis is trimmed to $3 \mathrm{~mm}^{3}$ fragments with micro-dissection scissors and adherent clotted blood is removed with forceps.

Following overnight incubation, tissues are infected with L. monocytogenes. The infection protocol described above is based on the Gentamicin protection assay used to study intracellular growth and spread of facultative intracellular bacteria ${ }^{16}$. Organ cultures in antibiotic-free medium are incubated with $L$. monocytogenes for $5 \mathrm{hr}$. Cultures are washed with sterile PBS to remove bacteria from the medium. Thereafter, Gentamicin is added to eliminate extracellular organisms. Prior literature from our laboratory used immunofluorescence and confocal microscopy to determine bacterial tissue localization at various timepoints after infection ${ }^{2}$. Organ cultures are rinsed in PBS, fixed in $4 \%$ paraformaldehyde, and either frozen-embedded in OCT medium and stored at $-80^{\circ} \mathrm{C}$, or paraffin-embedded and stored at room temperature. Tissue sections can be stained for immunofluorescence (IF) or immunohistochemical (IHC) analysis of bacteria and eukaryotic cell localization. Figure 2B is a representative IF image of a frozen section prepared from a 8.3 week villous organ culture at $72 \mathrm{hr}$ after infection. Note the heavy bacterial burden (green fluorescence) in the EVTs at the ends of the villous branches, and the relative lack of bacteria within the tree-lining syncytiotrophoblast (red fluorescence) layer. In previous work, we used similar IF staining to localize L. monocytogenes in villous organ cultures at different timepoints after infection. Over $72 \mathrm{hr}$, infection spread from EVT into sub-syncytial cytotrophoblasts and underlying villous stroma. Notably, the synyctiotrophoblast layer was resistant to infection ${ }^{2}$.

Representative light microscopic images of H\&E-stained paraffin-embedded decidual tissue sections prepared from 14.3 week specimens are shown in Figures 3B and 3C. This ECM-coated transwell membrane was embedded to show the organ culture in situ (Figure 3B). The image shows epithelial-lined glands (asterisk) and endothelial-lined vasculature (diamond) positioned heterogeneously within the decidual stromal cell compartment. Additionally, there are maternal immune cells scattered throughout the decidual stroma at a variable density. IHC and IF staining can be utilized to determine bacterial microanatomical localization, relative to specific resident immune cells. As an example, CD14 ${ }^{+}$ macrophages (green fluorescence) localize to the lining of vasculature and are scattered within the stroma (Figure 3D), while large aggregates of $L$. monocytogenes (red fluorescence) are observed predominantly in the decidual stroma at $48 \mathrm{hr}$ after infection (Figure 3D).

\begin{tabular}{|l|l|l|l|}
\hline Wash buffer & Collection medium & Villous medium & Decidual medium \\
\hline PBS & DMEM/F-12 with GlutaMAX & DMEM/F-12 with GlutaMAX & DMEM/F-12 with GlutaMAX \\
\hline Penicillin $100 \mathrm{IU} / \mathrm{ml}$ & Fetal Bovine Serum 2.5\% & Fetal Bovine Serum 20\% & Fetal Bovine Serum 2.5\% \\
\hline Streptomycin $100 \mu \mathrm{g} / \mathrm{ml}$ & Penicillin $100 \mathrm{IU} / \mathrm{ml}$ & Penicillin $100 \mathrm{IU} / \mathrm{ml}$ & $17 \beta$-estradiol $300 \mathrm{pg} / \mathrm{ml}$ \\
\hline Gentamicin $50 \mu \mathrm{g} / \mathrm{ml}$ & Streptomycin $100 \mu \mathrm{g} / \mathrm{ml}$ & Streptomycin $100 \mu \mathrm{g} / \mathrm{ml}$ & Progesterone $20 \mathrm{ng} / \mathrm{ml}$ \\
\hline Amphotericin B $1.25 \mu \mathrm{g} / \mathrm{ml}$ & Gentamicin $50 \mu \mathrm{g} / \mathrm{ml}$ & & \\
\hline & Amphotericin B $1.25 \mu \mathrm{g} / \mathrm{ml}$ & & \\
\hline
\end{tabular}

Table 1: Media recipes. Components and concentrations for preparation of Wash buffer, Collection medium, Villous medium, and Decidual medium. 

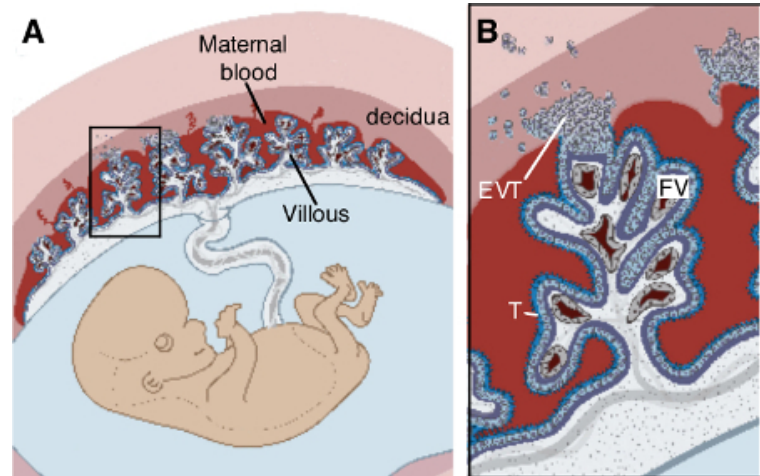

Figure 1. Placental structure. (A) Structure of feto-placental unit in maternal uterus, (B) Enlargement of box in (A) highlights that fetal trophoblast $(\mathrm{T})$ lined placental villi are bathed in maternal blood and anchored into the decidua by extravillous trophoblasts (EVT). Contained within villi are fetal vessels (FV), fibroblasts, and fetal macrophages. Modified from Zeldovich \& Bakardjiev ${ }^{22}$. Please click here to view a larger version of this figure.



Figure 2: Villous organ cultures - Representative gross and microscopic images. (A) Two terminal villous trees with a gestational age of 6 weeks, as viewed under a dissecting microscope. Note the "fluffy" ends (arrowheads) and prominent fetal vasculature coursing through the branches of the tree on the left that make this piece suitable for organ culture. (B) Immunofluorescence microscopy of organ culture (gestational age 8.3 weeks) $72 \mathrm{hr}$ post infection with Listeria monocytogenes, highlighting heavy bacterial burden [green] in extravillous trophoblasts. DAPI is shown in blue, and $\beta \mathrm{HCG}+$ syncytiotrophoblasts in red. Scale bars $=1 \mathrm{~mm}(\mathrm{~A}), 250 \mu \mathrm{m}(\mathrm{B})$. Please click here to view a larger version of this figure. 


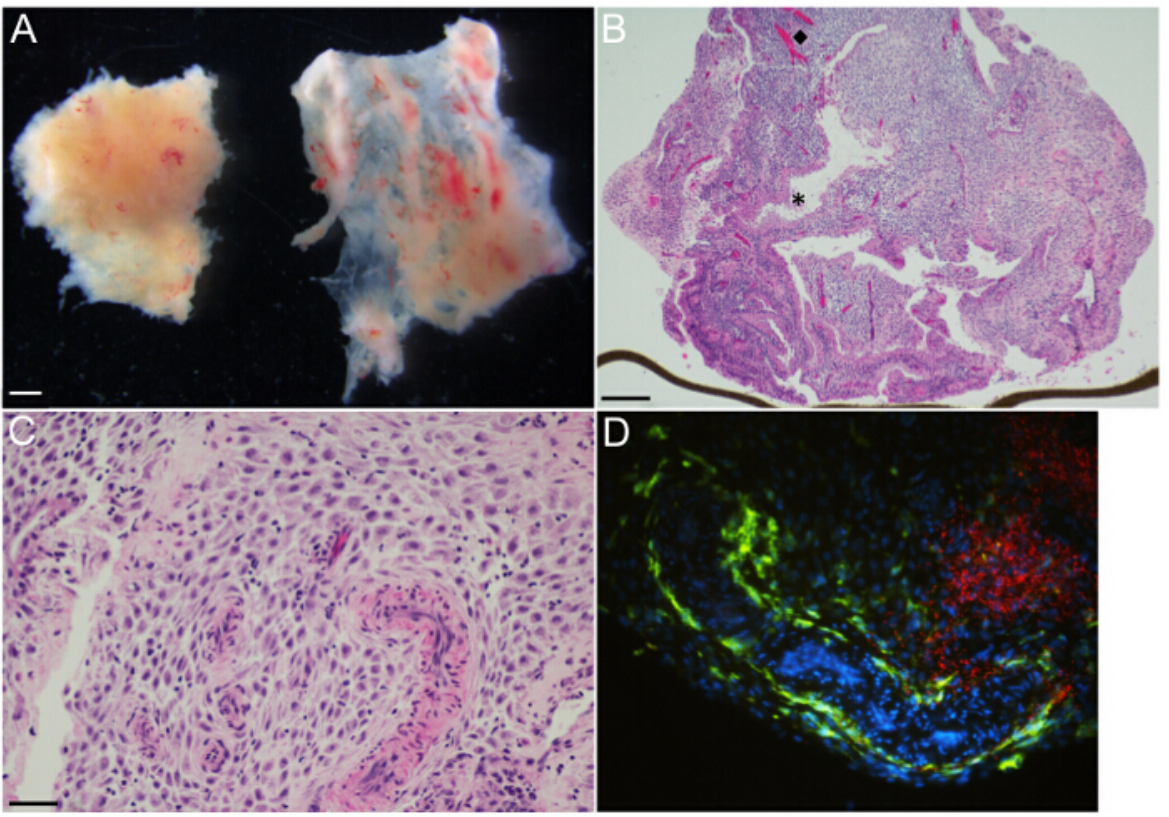

Figure 3: Decidual organ culture - Representative gross and microscopic images. (A) Decidua parietalis [left] and decidua capsularis [right] at gestational age 6 weeks, as viewed under a dissecting microscope. (B) H\&E-stained section of 14.3 week gestational age decidua parietalis organ culture shows glands and vasculature organized heterogeneously throughout decidual stroma. The (diamond, \#) and the (asterisk, ${ }^{*}$ ) highlight representative vessel and gland, respectively. Brown line at lower edge is transwell membrane, on edge. (C) H\&E-stained section of 14.3 week gestational age decidua parietalis organ culture at higher magnification demonstrates muscular-walled spiral arterioles embedded in decidual stroma. Maternal immune cells are small with dark round nuclei, and are irregularly distributed throughout the stroma. (D) Immunofluorescence microscopy of organ culture $48 \mathrm{hr}$ post infection with L. monocytogenes, highlighting large zones of bacteria [red] in decidual stroma, and maternal $\mathrm{CD} 14^{+}$macrophages [green] lining a tortuous vascular space and scattered in the stroma. Scale bars $=1 \mathrm{~mm}(\mathrm{~A})$, $250 \mu \mathrm{m}(\mathrm{B}), 50 \mu \mathrm{m}(\mathrm{C}$ and $\mathrm{D})$. Please click here to view a larger version of this figure.

Discussion

Inbred, transgenic and knockout mouse strains can serve in experimental systems to robustly test mechanisms. However, despite general conservation of core genetic material, the functional genomes of mice and humans demonstrate significant differences in regulatory elements ${ }^{23}$ It is not surprising, therefore, that promising preclinical studies in animal models are sometimes not recapitulated in human patients. The placenta shows very high interspecies diversity, thus rendering animal models less then ideal for the study of human disease ${ }^{24}$. Acknowledging both the notable differences in human and mouse immunology ${ }^{25}$, and the pronounced divergent evolution in placental anatomy, it is prudent to consider the use of ex vivo organ cultures of human gestational tissues for experimental investigation.

The descriptions, photographic images, and instructional video in this protocol instruct researchers on how to establish villous and decidual organ cultures on ECM-coated transwell tissue culture inserts. Advantages of this technique include the relative simplicity of the micro-dissection and mechanical support transwell system, particularly when compared to alternative methods such as 3D-embedded matrices or organ slice cultures. Suspension of the organ culture on membrane support allows for nutrient exchange at all tissue surfaces and viability is maintained for short-term culture ( $96 \mathrm{hr}$ for villous cultures, $72 \mathrm{hr}$ for decidual cultures). This technique allows for studying human placental biology at the tissue level, undeniably more biologically relevant than monolayer cell culture models.

The most critical step in this protocol is the micro-dissection of appropriate villous and decidual pieces for organ culture. Optimal pieces of tissue are demonstrated photographically (Figure 2A and Figure 3A) to aid researchers for whom visualization of gestational specimens is new. It is particularly important to select villous trees whose branches terminate in EVT columns, as these cells will migrate into the ECM and help anchor the villi to the membrane. For both types of organ cultures is helpful to minimize disturbances to the tissue during media changes by pipetting in a careful and unhurried manner.

This technique presents only minimal difficulties. On occasion, specimens show contamination. Contamination is usually bacterial (occasionally poly-microbial), and only becomes evident after 1-2 days in culture. Once contamination is observed, bleach should be applied, the culture discarded, and the tissue culture incubator sterilized to prevent a long-term problem. There are several possible ways contamination might arise: in utero infection, during surgery, during specimen harvest/processing, or during organ culture maintenance. The harvest and processing steps are the most likely time and place for contamination to be introduced. Thus, it is important to reduce the amount of time the specimen is manipulated during collection and micro-dissection. This will reduce specimen exposure to the ambient, non-sterile environment. Depending on laboratory setup, the dissecting microscope can be located inside the sterile tissue culture hood.

The histopathologic analysis of organ cultures after infection with the medically relevant pathogen $L$. monocytogenes, is shown here as one possible application of the protocol. Immunofluorescence localization of both bacteria and host immune cells after infection yields new insights into the human host response to pathogens. Decidual organ cultures might serve as a complementary ex vivo experiment technique to examine data generated from in vivo mouse infections, including intriguing reports showing defects in murine decidual macrophage defense functions ${ }^{26,27}$. Additionally, human organ cultures can be used in mixed-infection strategies, such as a competitive inoculum composed of several isogenic 
strains of $L$. monocytogenes. Competitive infection of organ cultures is a sensitive way to test the relevance of virulence factors within the human host. One constraint of this method is tissue viability, which is limited to short-term culture ( $96 \mathrm{hr}$ for villous cultures, $72 \mathrm{hr}$ for decidual cultures). This is ideal for infection with fast growing microbes such as $L$. monocytogenes, but longer cultures may be necessary for pathogens that take more time to establish and spread through tissue.

To reduce the global burden of pregnancy complications, research must focus on understanding the pathophysiology of the human maternal-fetal interface. Bacterial, fungal, and viral pathogens that cross from mother to fetus cause devastating complications of pregnancy and fetal infection. Controlled in vivo infection of laboratory animals is typically considered the gold standard for addressing how pathogens colonize and transit between organs ${ }^{28}$. Because placental anatomy varies markedly across mammalian species, it is of utmost importance to incorporate human tissue into research strategies. Human villous and decidual organ cultures are highly relevant model systems to investigate host-pathogen interactions. To study this vital yet poorly understood organ, researchers can take advantage of the abundance of otherwise discarded human placental and decidual specimens, using organ culture strategies such as described here.

\section{Disclosures}

The authors have nothing to disclose.

\section{Acknowledgements}

We are grateful to Cristina Faralla and David Lowe for helpful discussions. We acknowledge Mark Weinstein and San Francisco General Hospital Pathology Department for expert advice. This work was supported by National Institute of Health grants R01Al084928 and Burroughs Wellcome Fund 41259 to A.I.B; G.A.R. was supported by F32Al108195, Society for Pediatric Pathology Young Investigator Research grant, and University of California Partnerships for Faculty Diversity President's Post-doctoral Fellowship.

\section{References}

1. Pan, X., Yang, Y., \& Zhang, J. R. Molecular basis of host specificity in human pathogenic bacteria. Emerging microbes \& infections. 3, e23 (2014).

2. Robbins, J. R., Skrzypczynska, K. M., Zeldovich, V. B., Kapidzic, M., \& Bakardjiev, A. I. Placental syncytiotrophoblast constitutes a major barrier to vertical transmission of Listeria monocytogenes. PLoS pathogens. 6, e1000732 (2010).

3. Zhang, J. R. et al. The polymeric immunoglobulin receptor translocates pneumococci across human nasopharyngeal epithelial cells. Cell. $102,827-837$ (2000).

4. Ngampasutadol, J. et al. Human C4b-binding protein selectively interacts with Neisseria gonorrhoeae and results in species-specific infection Proceedings of the National Academy of Sciences of the United States of America. 102, 17142-17147 (2005).

5. Pishchany, G. et al. Specificity for human hemoglobin enhances Staphylococcus aureus infection. Cell host \& microbe. 8, 544-550 (2010).

6. Benirschke, K., Kaufmann, P., Baergen, R. N. Pathology of the Human Placenta. Springer-Verlag Berlin Heidelberg (2012).

7. Robbins, J. R., Zeldovich, V. B., Poukchanski, A., Boothroyd, J. C., \& Bakardjiev, A. I. Tissue barriers of the human placenta to infection with Toxoplasma gondii. Infection and immunity. 80, 418-428 (2012).

8. Shamir, E. R., \& Ewald, A. J. Three-dimensional organotypic culture: experimental models of mammalian biology and disease. Nature reviews. Molecular cell biology. 15, 647-664 (2014).

9. Hazan, A. D. et al. Vascular-leukocyte interactions: mechanisms of human decidual spiral artery remodeling in vitro. The American journal of pathology. 177, 1017-1030 (2010).

10. Dunk, C. et al. A novel in vitro model of trophoblast-mediated decidual blood vessel remodeling. Laboratory investigation; a journal of technical methods and pathology. 83, 1821-1828 (2003).

11. Hunkapiller, N. M., \& Fisher, S. J. Chapter 12. Placental remodeling of the uterine vasculature. Methods in enzymology. 445, 281-302 (2008).

12. Miller, J. M. et al. Guidelines for safe work practices in human and animal medical diagnostic laboratories. Recommendations of a CDCconvened, Biosafety Blue Ribbon Panel. MMWR Surveill Summ. 61 Suppl, 1-102 (2012).

13. Lash, G. E. et al. Low oxygen concentrations inhibit trophoblast cell invasion from early gestation placental explants via alterations in levels of the urokinase plasminogen activator system. Biol Reprod. 74, 403-409 (2006).

14. Wang, N., Strugnell, R., Wijburg, O., \& Brodnicki, T. Measuring bacterial load and immune responses in mice infected with Listeria monocytogenes. Journal of visualized experiments : JoVE. (2011).

15. Palmer, M. E., Watson, A. L., \& Burton, G. J. Morphological analysis of degeneration and regeneration of syncytiotrophoblast in first trimester placental villi during organ culture. Hum Reprod. 12, 379-382 (1997).

16. Elsinghorst, E. A. Measurement of invasion by gentamicin resistance. Methods in enzymology. 236, 405-420 (1994).

17. Kucherenko, M. M. et al. Paraffin-embedded and frozen sections of Drosophila adult muscles. Journal of visualized experiments : JoVE. (2010).

18. Croy, B. A., Yamada, A. T., DeMayo, F. J., Adamson, S. L. The Guide to Investigation of Mouse Pregnancy, 1st Edition. (2014).

19. JoVE Science Education Database. General Laboratory Techniques. Histological Sample Preparation for Light Microscopy. JoVE, Cambridge, MA (2016).

20. Troy, T. C., Arabzadeh, A., Enikanolaiye, A., Lariviere, N., \& Turksen, K. Immunohistochemistry on paraffin sections of mouse epidermis using fluorescent antibodies. Journal of visualized experiments : JoVE. (2008).

21. Chi, V., \& Chandy, K. G. Immunohistochemistry: paraffin sections using the Vectastain ABC kit from vector labs. Journal of visualized experiments : JoVE. e308 (2007).

22. Zeldovich, V. B., \& Bakardjiev, A. I. Host defense and tolerance: unique challenges in the placenta. PLoS pathogens. 8, e1002804 (2012).

23. Yue, F. et al. A comparative encyclopedia of DNA elements in the mouse genome. Nature. 515, 355-364 (2014).

24. Robbins, J. R., \& Bakardjiev, A. I. Pathogens and the placental fortress. Current opinion in microbiology. 15, 36-43 (2012).

25. Mestas, J., \& Hughes, C. C. Of mice and not men: differences between mouse and human immunology. J Immunol. 172, 2731-2738 (2004). 
26. Redline, R. W., Shea, C. M., Papaioannou, V. E., \& Lu, C. Y. Defective anti-listerial responses in deciduoma of pseudopregnant mice. The American journal of pathology. 133, 485-497 (1988).

27. Redline, R. W., McKay, D. B., Vazquez, M. A., Papaioannou, V. E., \& Lu, C. Y. Macrophage functions are regulated by the substratum of murine decidual stromal cells. The Journal of clinical investigation. 85, 1951-1958 (1990).

28. Bakardjiev, A. I., Theriot, J. A., \& Portnoy, D. A. Listeria monocytogenes traffics from maternal organs to the placenta and back. PLoS pathogens. 2, e66 (2006). 